NASA/TM-1999-206569

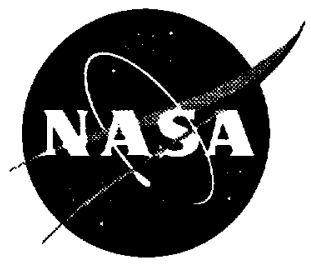

\title{
Flight Test of an Adaptive Configuration Optimization System for Transport Aircraft
}

Glenn B. Gilyard, Jennifer Georgie, and Joseph S. Barnicki

Dryden Flight Research Center

Edwards, California

National Aeronautics and

Space Administration

Dryden Flight Research Center

Edwards, California 93523-0273

January 1999 


\section{NOTICE}

Use of trade names or names of manufacturers in this document dors not constitute an official endorsement of such products or manufacturers, either expressed or impli 2 , by the National Aeronautics and Space Administration.

Available from the following:

NASA Center for AeroSpace Information (CASI)

7121 Standard Drive

Hanover, MD 21076-1320

(301) 621-0390
National Technical Information Service (NTIS)

5285 Port Royal Road

Springfield, VA 22161-2171

(703) $487-4650$ 


\title{
FLIGHT TEST OF AN ADAPTIVE CONFIGURATION OPTIMIZATION SYSTEM FOR TRANSPORT AIRCRAFT*
}

\author{
Glenn B. Gilyard, ${ }^{\dagger}$ Jennifer Georgie, ${ }^{\ddagger}$ and Joseph S. Barnicki ${ }^{\S}$ \\ NASA Dryden Flight Research Center \\ P.O. Box 273 \\ Edwards, California 93523-0273
}

\begin{abstract}
$\underline{\text { Abstract }}$
A NASA Dryden Flight Research Center program explores the practical application of real-time adaptive configuration optimization for enhanced transport performance on an L-1011 aircraft. This approach is based on calculation of incremental drag from forcedresponse, symmetric, outboard aileron maneuvers. In real-time operation, the symmetric outboard aileron deflection is directly optimized, and the horizontal stabilator and angle of attack are indirectly optimized. A flight experiment has been conducted from an onboard research engineering test station, and flight research results are presented herein. The optimization system has demonstrated the capability of determining the minimum drag configuration of the aircraft in real time. The drag-minimization algorithm is capable of identifying drag to approximately a one-drag-count level. Optimizing the symmetric outboard aileron position realizes a drag reduction of 2-3 drag counts (approximately 1 percent). Algorithm analysis of maneuvers indicate that two-sided raised-cosine maneuvers improve definition of the symmetric outboard aileron drag effect, thereby improving analysis results and consistency. Ramp maneuvers provide a more even distribution of data collection as a function of excitation deflection than raised-cosine maneuvers provide. A commercial operational system would require airdata calculations and normal output of current inertial navigation systems; engine pressure ratio measurements would be optional.
\end{abstract}

* Patent pending.

${ }^{\dagger}$ Glenn B. Gilyard, Aerospace Engineer, (805) 258-3724.

$\ddagger_{\text {Jennifer Georgie, Student Trainee, Engineering, Texas }}$ A\&M University.

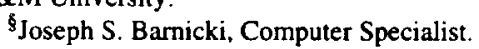

Copyright (C) 1998 by the American Institute of Aeronautics and Astronautics, Inc. No copyright is asserted in the United States under Title 17, U.S. Code. The U.S. Government has a royalty-free license to exercise all rights under the copyright claimed herein for Governmental purposes. All other rights are reserved by the copyright owner.

\section{$\underline{\text { Nomenclature }}$}

APO adaptive performance optimization

$C_{L} \quad$ coefficient of lift

$C_{D} \quad$ coefficient of drag

$C_{L} @ \min C_{D} C_{L}$ at minimum $C_{D}$

$C_{D_{h}} \quad$ coefficient of drag due to altitude

$C_{D_{M}} \quad$ coefficient of drag due to Mach

$C_{D_{M}{ }^{2}} \quad$ coefficient of drag due to $\mathrm{Mach}^{2}$

$C_{D_{0}} \quad$ zero-lift drag coefficient

GPS global positioning system

h altitude, $\mathrm{ft}$

INS inertial navigation system

$K_{1}, K_{2} \quad$ drag equation coefficients

$M \quad$ Mach number

RBNB Ring Buffered Network Bus ${ }^{\text {TM }}$

RETS research engineering test station

$t \quad$ time, sec

$\delta_{a_{o p t}} \quad$ optimal (minimum drag) symmetric outboard aileron position, deg

$\delta_{a_{\text {opt }} \text { comd }}$ commanded optimal symmetric outboard aileron position, deg

$\delta_{a_{\text {sym }}} \quad$ symmetric outboard aileron position, deg $\Delta \quad$ change

$\sigma \quad$ root mean square

\section{Introduction}

Aircraft efficiency is an important factor for aircraft manufacturers and airline operators. For manufacturers, operating costs are an important element in maintaining and increasing market share of aircraft sales. For airlines, operating costs relate directly to profit. Fuel 
costs can approach 50 percent of the operating expense for some types of modern, wide-body, long-range transports. "11 A 1-percent reduction of fuel consumption can produce savings of as much as $\$ 140,000$ each year for each aircraft.

In addition to these direct savings, for aircraft that are at maximum takeoff weight, less fuel at takeoff allows additional payload. Revenue from $1 \mathrm{lbm}$ of payload is worth as much as 30 times more than the cost of $1 \mathrm{lbm}$ of fuel; thus, benefits of a 1-percent drag reduction for each aircraft can be $\$ 4,000,000$ or more each year. For aircraft that are at maximum fuel but less than maximum takeoff weight, approximately $3 \mathrm{lbm}$ of payload can be added for every $1 \mathrm{lbm}$ of fuel not required. The additional revenues from this scenario are as much as 90 times more than the cost of fuel; thus, benefits of a 1-percent drag reduction for each aircraft can be $\$ 12,000,000$ or more each year. Increased revenue benefits can likely exceed the benefit of reduced fuel cost for the wide-body fleets of some airlines. The reduced fuel consumption also produces equivalent reductions in atmospheric gas emissions, which is becoming an increasingly important environmental issue.

Significant potential exists for improving aircraft efficiency through the use of variable geometry and, more specifically for transport aircraft, variable camber. The F-111 Mission Adaptive Wing program demonstrated the benefits of applying variable-camber geometry to optimize various flight conditions for fighter configurations, including cruise performance. ${ }^{2}$ Design work is ongoing for implementation of variable camber into future transport aircraft. ${ }^{3}$

All current transport aircraft have latent potential for varying degrees of variable-camber control. For fly-bywire aircraft, the potential can be realized relatively easily with software modifications; whereas for aircraft with mechanical controls, modifications in the flight control system hardware are also required. The most obvious control surfaces that can be used to implement variable camber on transport aircraft include outboard and inboard ailerons, flaps, the horizontal stabilizer, and the elevator.

The NASA Dryden Flight Research Center (Edwards, California) is involved in an adaptive performance optimization (APO) research program to develop concepts and validate technologies for drag reduction on

\footnotetext{
This cost does not include fleet ownership and overhead expenses, which together are nearly equal to the operating expenses.
}

transport ai craft. The APO program approach is to adaptively optimize available, redundant, variable geometry to minimize the net aircraft drag. For the current rese arch program, symmetric outboard aileron deflections are used in a variable-camber-type mode to optimally recamber the wing to minimize drag for all aircraft conigurations and flight conditions. Realizing small performance benefits $(0.5-2.0$ percent $)$ is challenging. The proposed drag-minimization algorithm uses measurement-based optimal control for performance improvement using variable geometry of the wing.

The modifications required to support the APO program included the addition of an actuator on each wing. These actuators drive the outboard ailerons symmetricaly to provide some variable-camber capability.

A review of related variable-camber and optimization technology issues and simulation evaluations of the proposed optimization algorithm has previously been presented. ${ }^{4}$ Details of the modifications to the L-1011 test bed arcraft and a proposed approach for an operational implementation of the optimization system have also been published. 5

This paper summarizes the results of three research flights. Two flights (baseline) collected data from forced-response maneuvers for postflight analysis and development of a real-time adaptive configuration optimizatio I algorithm. The third flight (real-time) demonstrat $d$ the first-ever operation of an in-flight adaptive configuration optimization algorithm for performance improvement. Background material and a brief overvew of the research flight systems are also given. The discussion includes data analysis issues regarding the low signal-to-noise ratio of small incremental drag estimates. Various maneuvers and variations iı parameters defining the maneuvers are also discussed. Representative maneuvers and optimization results are resented.

Use of tride names or names of manufacturers in this document does not constitute an official endorsement of such prodicts or manufacturers, either expressed or implied, by the National Aeronautics and Space Administra ion.

\section{Background: Transport Performance Optimization}

Current iubsonic transport design for cruise flight results in a point-design aerodynamic configuration. By 
necessity, the final configuration is a major compromise among a multitude of design considerations. Additionally, the final design provides near-optimal performance for specifically defined flight profiles and results in the aircraft flying at its best performance design condition very seldom or only by chance. In the cruise configuration, no additional configuration changes are available to optimize performance for the vast range of constraints. Such operational and external constraints include air-traffic-control directives (speed and altitude), loading (cargo and fuel), center of gravity, flight length, variations in manufacturing, aging, and asymmetries.

No aircraft currently has an adaptive configuration optimization system. However, manual configuration optimization is attempted on all transport aircraft during takeoff, approach, and landing situations when flaps are used to improve, or "optimize," low-speed lift requirements. Adaptive configuration optimization is the natural extension for drag reduction at cruise flight of what is currently done manually to improve lift characteristics during low-speed flight.

Aircraft currently use the flight management system as the main tool to obtain some degree of in-flight performance "optimization." The term "optimization" is used widely and loosely, and, in a discussion of this nature, consistency and the ability to distinguish the different types of optimization are important. The above-mentioned flight management system "optimization" is more accurately referred to as "trajectory optimization" (generally optimizing altitude at a fixed Mach number) and is based on models of predicted and flight characteristics for one specific aircraft generated early in the flight test program.

The differences among models and the actual aircraft should be small, but because of inaccuracies in aerodynamic and engine models and actual aircraft changes over time, differences between the flight management system model and the actual aircraft could be significant. If the actual performance-related characteristics of a specific aircraft can be determined in flight, that information can be used to obtain actual, true trajectory optimization, which is better than benefits available with a preprogrammed flight management system. These trajectory optimization benefits are separate from configuration optimization benefits (although not independent); however, the two optimization processes are complementary.

Many issues enter into the subject of configuration optimization for performance enhancement of subsonic transport aircraft. Foremost, the potential for optimization must exist, which implies redundant control effector capability (for instance, more than one means of trimming the forces and moments to obtain a steady-state flight condition). Most aircraft have latent capability in this area, although taking advantage of this capability from hardware and software aspects can be complex. The range of controls or variables include elevator, horizontal stabilizer, outboard aileron, inboard aileron, rudder, center of gravity, and thrust modulation; the benefits have previously been discussed. ${ }^{4}$

In addition, performing optimization from a condition that is already fine-tuned (based on wind-tunnel and flight testing) requires high-quality instrumentation and comprehensive analytical techniques to enable estimation of small drag changes in an unsteady environment. Instrumentation available on modern transports should be adequate for performing adaptive optimization.

\section{Test Bed Aircraft Description}

An L-1011 (Lockheed Corporation, Burbank, California) aircraft (fig. 1) was selected as the test bed and modified for the APO flight experiment. The L-1011 aircraft is representative of the general class of widebody transports capable of long-range cruise fight. Aircraft availability and cost dictated this aircraft over other wide-body transports.

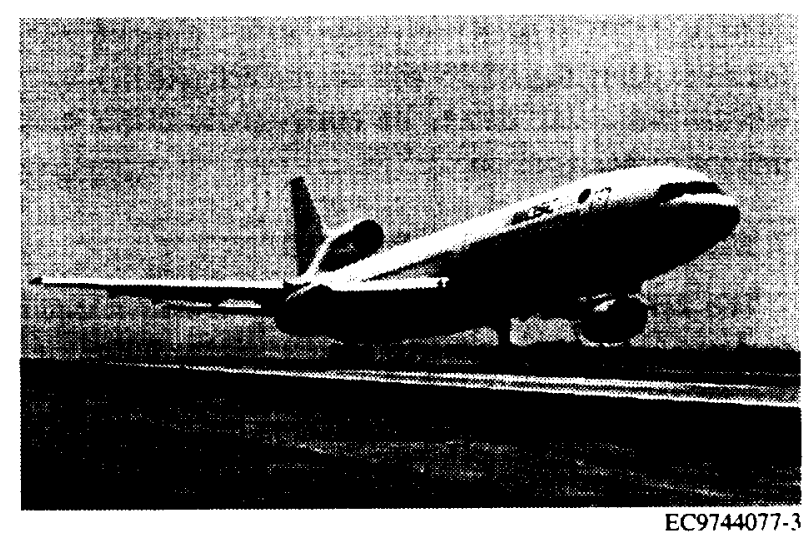

Figure 1. Modified L-1011 test bed aircraft.

\section{Test Aircraft}

The test aircraft is a L-1011-100 model that was previously modified to launch satellites using various models of the Pegasus ${ }^{\circledR}$ (Orbital Sciences Corporation, Dulles, Virginia) rocket. The aircraft is powered by three 
RB-211-22B (Rolls Royce, Inc., Derby, England) highbypass turbofan engines. The empty weight and maximum gross takeoff weight of the modified aircraft (without the Pegasus ${ }^{\circledR}$ ) are 220,000 and $474,000 \mathrm{lbm}$, respectively. The aircraft has a cruise range of approximately $4000 \mathrm{mi}$ at Mach 0.84 and a maximum operating Mach of 0.90 . The research flights were supported and flown by Orbital Sciences Corporation under contract to NASA.

\section{Test Bed Modifications}

Aircraft modifications necessary to support the APO experiment consisted of the following:

- the addition of a research engineering test station (RETS).

- the addition of an actuator, one on each wing, to drive the outboard ailerons symmetrically.

- the addition of a trailing-cone system to obtain true static pressure.

- a connection into the basic data system of the ship to obtain engine, control surface, and other measurements.

- the addition of an embedded global positioning system/inertial navigation system (GPS/INS).

- the addition of a state-of-the-art airdata computer.

Although the INS was embedded with a GPS, the additional GPS-related parameters are not a requirement for APO. Only the RETS and aileron actuation systems will be discussed; details on other modifications have previously been presented. ${ }^{5}$

\section{Research Engineering Test Station}

The RETS was designed to be a very flexible research tool and has many capabilities, including the following:

- generation of forced-excitation signals to drive the outboard ailerons.

- position control for the outboard aileron actuators.

- data calibration, collection, and storage.

- data and analysis displays.

- real-time analysis.

- display of variables and calculated parameters.

- automatic feedback control and optimization of the outboard ailerons.
- monitoring system health.

- commu uications with the pilot station.

The forced-xcitation set consists of steps, ramps, sine waves, and raised-cosine waves.

\section{Symmetric Outboard Aileron Actuation}

The L-1011-100 aileron control system is fully mechanical; the outboard aileron is commanded from the inboard aileron using pushrods and cables. The approach taken to provide symmetric control to the outboard ailerons was to modify the rod coming out of the inboard aileron that drives the outboard aileron. The modification consisted of replacing the rod with a lowbandwidth, constant-speed, electric actuator with end fittings identical to the rod being replaced. This modification provides for an adjustable rod length, thus permitting independent commands to be summed for each outboard aileron. The control of the actuator position requires position feedback control and is performed by software.

The output position of the modified rod is thus the sum of inboard aileron position (of which the pilot has full command) plus the RETS command sent to the modified actuator rod. In the research application, the option of hiving the outboard aileron follow a desired excitation command and not contain or be "contaminated" by the inboard aileron command is available. This availability is achieved by measuring the inboard aile:on position and subtracting this signal from the desired excitation signal. This signal, when summed with the inboard aileron position, is equal to the desired excitation $\mathrm{c}$ mmand.

\section{Reil-Time Flight Test Operations}

Forced excitation is required to identify incremental drag effects The requirement for forced excitation must be consistent with the additional requirement that neither has dling nor ride qualities are noticeably impacted, $w$ hich in turn dictates the range of excitation frequencies and amplitudes.

The AP( ) flight experiment only considered the explicit control of the outboard ailerons; the stabilator and angle- $\mathrm{f}$-attack changes are implicitly controlled through the altitude-hold autopilot. When the pilot applies pow ar to the APO system, the test conductor has control ove: the actual surface position of the outboard ailerons. The experimental APO system has the following test setup and actuator feedback control capabilities 
- A bias can be added to either or both outboard ailerons to control them symmetrically, independent of either pilot or autopilot inputs. In the case where either pilot or autopilot inputs are required for roll axis control, the deflections of the inboard ailerons will be increased as required to account for the "loss" of outboard aileron control in the roll axis.

- Step, ramp, sine, or raised-cosine excitation commands are available. The magnitude, frequency, and maneuver duration is selectable as required.

- The maximum commandable actuator position and rate limit sent to the outboard aileron is selectable and controlled by software.

- The relay hysteresis characteristics that control the drive commands sent to the actuator for position feedback control of the actuator are selectable by software. Hardware-in-the-loop tuning of the actuator feedback control loop was required to minimize actuator activity.

\section{Flight Experiment Operation}

The test conductor selects the test setup options described in the previous section. The desired flight condition is stabilized by the pilot and autopilot. Altitude is controlled by the altitude-hold mode of the autopilot. Ideally, an autothrottle mode would be used to control Mach number, but because the test bed aircraft does not have that mode, Mach number can be allowed to vary or can be controlled by the pilot. Any Machnumber variations are compensated for in the analysis.

When the test conductor determines flight conditions have stabilized, the excitation function is commanded. The outboard aileron movement causes small drag changes. These drag changes are desired to be on both sides of the minimum to ensure identification of the minimum drag condition (to be discussed in the next section).

For the baseline flights, data were collected onboard and analyzed postflight. For the real-time flights, data were collected throughout the maneuver and the dragminimization analysis was performed in parallel. When the minimum-drag geometry is identified, the outboard ailerons are then commanded to that optimal position. The most obvious way to take advantage of the drag reduction is to continue flying at the same desired flight conditions but at reduced fuel flows. An alternate use of the reduced drag is to increase the cruise speed at the same fuel flow setting. Other variations on how the benefits of reduced drag can be utilized also exist.

\section{Drag-Minimization Algorithm}

To provide an effective optimization algorithm, estimation of incremental drag changes of 1 percent or less are required. Although absolute drag measurements of this accuracy are only obtainable with very detailed analysis and precise engine modeling, incremental drag values in this range are readily achievable.

The postflight and real-time optimization algorithm is based on identification of unknown drag equation coefficients from a smooth, low-frequency forcedresponse maneuver. The analysis assumes steady-state flight; therefore, the forced-excitation maneuver must be sufficiently slow so that quasi-equilibrium is always maintained and no significant dynamic effects exist.

The analysis requires accurate linear and angular displacement, velocity, and acceleration measurements (such as from an INS) and accurate airdata information. Angle-of-attack estimations are calculated from inertial measurements and airdata. Thrust is estimated from a representative steady-state engine model as a function of engine pressure ratio, Mach number, and altitude. The lift and drag equations are then used to calculate the coefficient of lift, $C_{L_{5}}$, and the coefficient of drag, $C_{D}$, as a function of time. ${ }^{5}$

The following equation is an expansion of $C_{D}{ }^{5}$ that is a function of available parameters $\left(C_{D}, C_{L}, \delta_{a_{s y m}}\right.$, $\Delta M, \Delta M^{2}$, and $\left.\Delta h\right)$ and unknown drag coefficients $\left(C_{D_{0}}, K_{I}, C_{L} @ \min C_{D}, K_{2}, \delta_{a p i}, C_{D_{M}}, C_{D_{M}{ }^{2}}\right.$, and $C_{D_{h}}$ ) that includes a quadratic representation of drag due to symmetric outboard aileron deflection.

$$
\begin{aligned}
C_{D}= & C_{D_{0}}+K_{l}\left[C_{L}-C_{L @ \min } C_{D}\right]^{2} \\
& +K_{2}\left(\delta_{a_{s y m}}-\delta_{a_{o p t}}\right)^{2}+C_{D_{M}} \Delta M \\
& +C_{D_{M^{2}}} \Delta M^{2}+C_{D_{h}} \Delta h
\end{aligned}
$$

This equation results in a set of equations (equal in size to the number of data samples collected) that are then solved using regression analysis.

The $C_{D}$ formulation is not unique; the important element is that the first-order effects of aileron-induced 
drag be represented in the $C_{D}$ equation in a plausible manner. Care should be taken not to over-parameterize the problem; independence of the various estimates must be maintained to provide meaningful results.

Simulation results ${ }^{4}$ confirm that the analysis procedure is insensitive to a wide range of algorithm variables such as a priori estimates of aircraft $C_{L}$ as a function of $C_{D}$, measurement bias and resolution effects, and thrust model accuracy.

\section{Flight Results}

Four flights have been conducted to date: one flight to check out the research systems functionality, two research flights to collect baseline data for postflight analysis and algorithm development, and one research flight for demonstration of a real-time adaptive configuration optimization algorithm. The functional flight demonstrated and verified proper operation of all the experiment-related command and control functions and the instrumentation system. The two baseline flights encountered significant turbulence; few data were collected in smooth atmospheric conditions. The realtime research flight primarily experienced smooth atmospheric conditions and had only very infrequent low levels of turbulence. The three research flights each lasted approximately $8 \mathrm{hr}$.

\section{Baseline Postflight Analysis}

The two baseline research flights consisted of collecting aircraft response data to forced-excitation maneuvers. The objectives of the postflight analysis were to refine the analysis algorithm; ${ }^{5}$ evaluate the parameters of the excitation maneuver (for example, amplitude and frequency); evaluate various maneuver types; and demonstrate algorithm results.

The identification process, which determines the unknowns in the expanded $C_{D}$ equation such as the optimal symmetric aileron setting, consists of a set of static equations. Because any arbitrary control surface motion will introduce dynamics, the maneuver should be very slow so as to minimize dynamic response. The slow maneuver is also intended to minimize any coupling between the maneuver excitation and the control surface commands of altitude- and Mach-hold autopilot modes used to constrain deviations in altitude and Mach number.

The aircraft response characteristics during the maneuver should be nearly indistinguishable from normal cruise flight. A raised-cosine maneuver satisfies the requirem ents stated above and appears to be an ideal maneuver be zause of the smooth characteristics of it and its derivatives. Simulation studies indicated that a period of a minir lum $300 \mathrm{sec}$ would meet the above requirement.

\section{Raised-Cssine Excitation}

Figure 2 shows a representative maneuver performed at Mach 0.84 and an altitude of $35,000 \mathrm{ft}$. The outboard aileron excitation period was $400 \mathrm{sec}$ and the amplitude was $-8^{\circ}$ trailng edge down; 2 min of data were obtained both at the beginning and end of the run with no excitation input. The altitude-hold mode constrains altitude to $:: 10 \mathrm{ft}$ throughout the maneuver. The test aircraft did not have an autothrottle mode to control Mach number; therefore, the Mach number varied approximately 0.01 peak-to-peak. This variation is accounted for in the analysis by including Mach and altitude terms in the expanded $C_{D}$ equation.

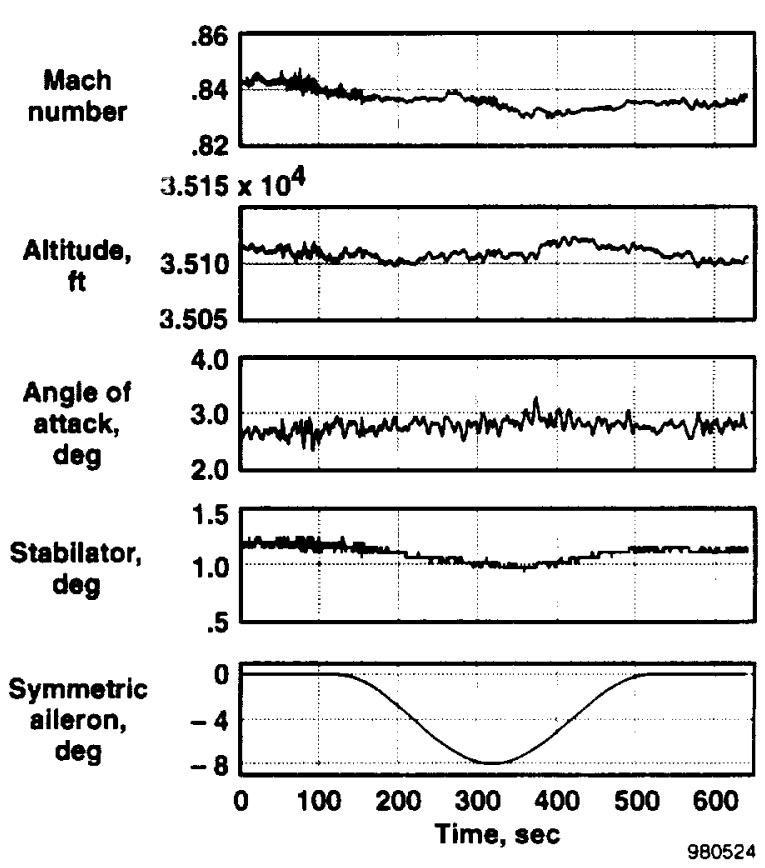

Figure 2. Flight response to a raised-cosine excitation of the outboarc ailerons.

The horiz ontal stabilator and angle-of-attack changes required to naintain the constrained altitude condition are interesting to note. The horizontal stabilator change is indirectly taken into account in the analysis, whereas the angle of attack is an analysis variable. The challenge is to identify the optimal aileron position from data that have a very ow signal-to-noise ratio. 


\section{Data Filtering}

Figure 3 shows the output of the optimization analysis, using the data of the previous time history. The $\Delta C_{D}$ represents only the effect due to the symmetric aileron deflection, and the smooth fairing is the best fit of the data (assuming the variation of $C_{D}$ due to symmetric aileron is quadratic). The data noise-to-signal level is much greater than an order of magnitude and although the fit is not poor, concluding the fit is good is difficult. The "noise" is primarily a result of the longitudinal and normal acceleration measurements, and this very low signal-to-noise ratio makes identification of small, incremental $C_{D}$ estimates challenging. The fit yields a minimum $\Delta C_{D}$ of -0.000236 at $\delta_{a_{\text {opt }}}=-4.5$.

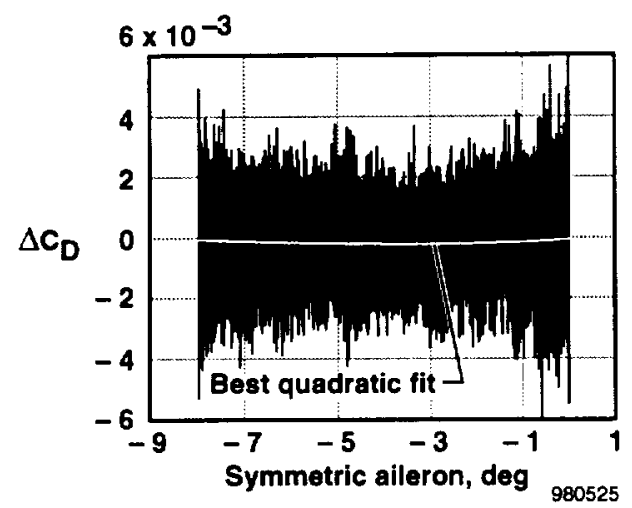

Figure 3. Variation of incremental drag with symmetric outboard aileron deflection for a one-sided raised-cosine maneuver (no filtering, period $=400 \mathrm{sec}, \sigma=0.0012$ ).

To improve the graphical comparison of the data and the fit (and thus improve confidence in the analysis), various filtering schemes were explored. Because the frequency content of the data of interest is very low (period $=400 \mathrm{sec}$ ), the resulting low-pass filter, in addition to filtering what is normally thought of as noise, was also designed to remove aircraft short-period dynamics (which are still well-separated from the frequency of interest). Performing the analysis with this filter produces the $\Delta C_{D}$ as a function of $\delta_{a_{s y m}}$ (fig. 4) (note that an order-of-magnitude difference exists on the ordinates of figures 3 and 4). The signal-to-noise level of $\Delta C_{D}$ as a function of $\delta_{a s y m}$ is dramatically improved, and the fit of the data appears reasonable. The fit yields a minimum $\Delta C_{D}$ of -0.000248 at $\delta_{a_{\text {opt }}}=-4.5$; these results compare very favorably with those of figure 3 and indicate that the filter did not exclude data of interest.

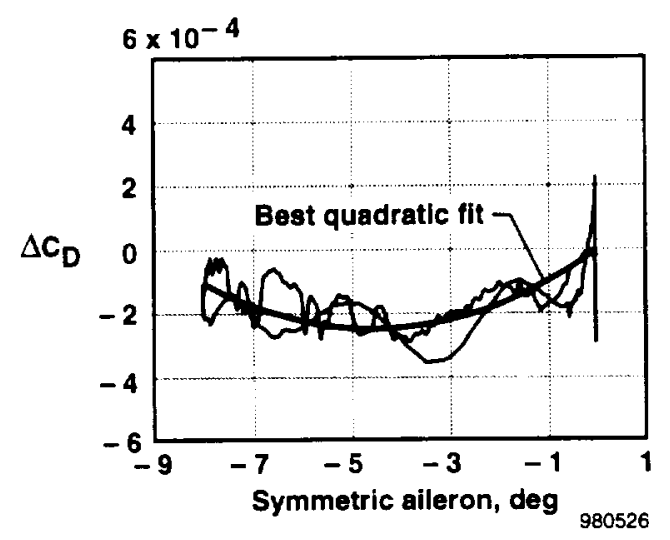

Figure 4. Variation of incremental drag with symmetric outboard aileron deflection for a one-sided raised-cosine maneuver (data filtered, period $=400 \mathrm{sec}$, $\sigma=0.000076$ ).

\section{Two-Sided Raised-Cosine Excitation}

In an effort to empirically gain insight into the effect of excitation type, a number of different excitation shapes and durations were explored. A two-sided raisedcosine maneuver that consists of combining a negative and a positive raised-cosine maneuver has the advantage of providing excitation on both sides of the trimmed aileron position. The idea is that a wider spread in the $\delta_{a_{\text {sym }}}$ command will improve the quality and consistency of the analysis results. The second portion of the maneuver is started before completion of the first to produce a nearly constant excitation command rate in going from the first peak to the second peak.

Another parametric study involved the use of the twosided raised-cosine maneuver with a fixed amplitude with periods of $300,200,150,100,50$, and $25 \mathrm{sec}$. Because of the blending of the two pieces, the maneuver looks somewhat like a sine wave. However, the period applies to each portion, and the total excitation time is less than double the period because overlap of the two excitation portions exists. Figure 5 shows the variation of $\Delta C_{D}$ as a function of $\delta_{a_{s y m}}$ for a two-sided raisedcosine maneuver. A period of $150 \mathrm{sec}$ was used, and the benefit of providing excitation in both directions has clearly contributed to a more precise definition of the $\Delta C_{D}$ variation. 


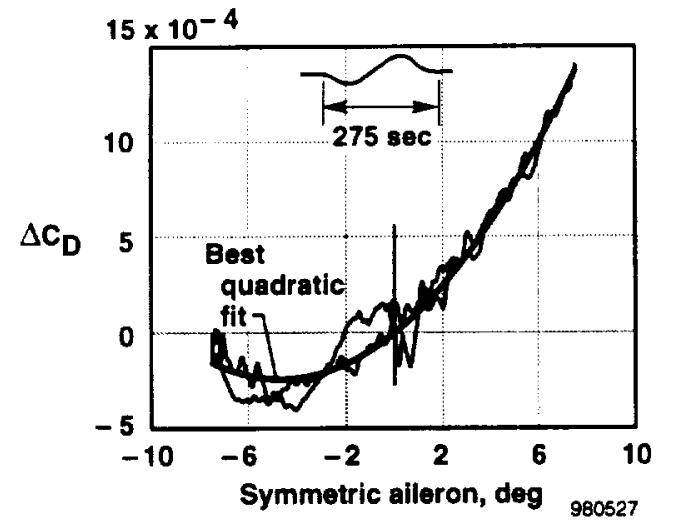

Figure 5. Variation of incremental drag with symmetric outboard aileron deflection for a two-sided raised-cosine maneuver (data filtered, period $=150 \mathrm{sec}$, $\sigma=0.000125$ ).

Figure 6 shows a summary of all six maneuvers. The three maneuvers with excitation periods of 300,200 , and $150 \mathrm{sec}$ appear to provide very consistent results; the optimal (minimum drag) $\delta_{a p t}$ ranges from $-4.6^{\circ}$ to $-5.3^{\circ}$, and the average drag reduction is $205 \pm 30 \mathrm{lbf}$. The 100-sec maneuver is quite fast, and the analysis predicts an overly optimistic drag reduction at an unrealistic $\delta_{a p t}$ deflection. The two maneuvers with periods of 50 and $25 \mathrm{sec}$ are too fast and clearly violate the assumption that all the data collected during the maneuver are nearly steady-state.

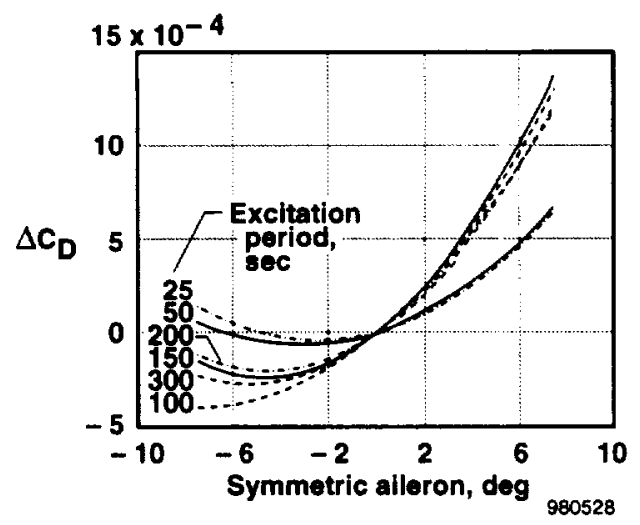

Figure 6. Comparison of variation of incremental drag with symmetric outboard aileron deflection for twosided raised-cosine maneuvers with periods of 300,200 , $150,100,50$, and $25 \mathrm{sec}$ (data filtered).

\section{Excitation Analysis}

Although the smoothness of the raised-cosine maneuver is a desirable characteristic, a large variation exists in the ate at which the aileron is commanded, and this feature rnay introduce dynamics into the maneuver. Figure 7 s lows the histogram of a raised-cosine maneuver th it illustrates the disproportionate amount of time the excitation is at or near the extremes deflection as compared with the time spent traversing between these extremes.

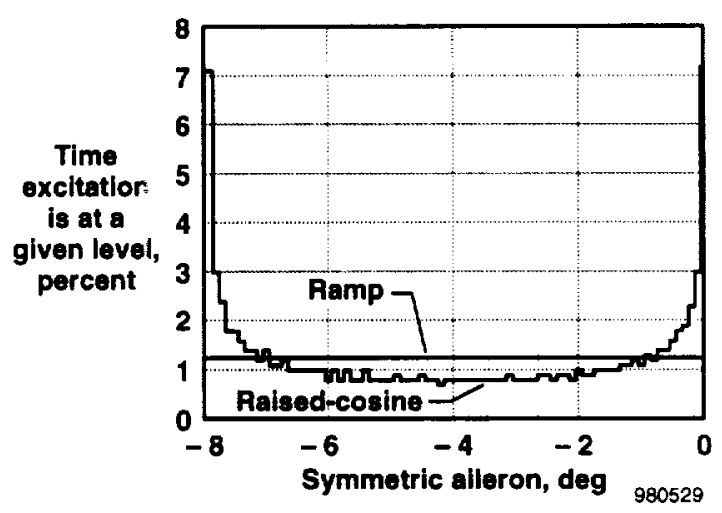

Figure 7. Histogram of raised-cosine and ramp excitation functions.

Although a ramp has a rate discontinuity both starting and stoppin!, if a low rate is selected, the discontinuity should not ntroduce noticeable dynamic effects. The histogram for a constant-rate ramp covering the same aileron range as the raised-cosine maneuver of figure 7 would have a constant magnitude of 1.25 percent of the time spent a: each $0.1^{\circ}$ of aileron change. Although that magnitude nay not seem like much, approximately 50 percent nore time is spent traversing each $0.1^{\circ}$ of aileron deflction with the ramp than during the fastest portions of the raised-cosine maneuver. A rampexcitation nianeuver would collect significantly more data during the traversing period than the raised-cosine maneuver, t tus providing a better distribution of data over the ran se of excitation.

Figure 8 shows the variation of $\Delta C_{D}$ as a function of $\delta_{a_{\text {sym }}}$ for a ramp excitation. The excitation ranged from $0^{\circ}$ to $-6^{\circ}$ in $120 \mathrm{sec}$ for a rate of $0.05 \mathrm{deg} / \mathrm{sec}$. The fit describe: the gross trend fairly well, although the minimum $\left(-6^{\circ}\right)$ indicates a larger trailing-edge-down drag minim sm than most previous results. Looking at 
only the data, the minimum appears to be between -3.5 and -5.0 . This difference between the fit minimum and the apparent data minimum indicates that assuming a quadratic variation of drag with respect to symmetric aileron may not be ideal. However, the fact that the data are mostly on one side of the minimum and the relatively high noise-to-signal ratio in the unfiltered data can account for significant variation in the optimization results.

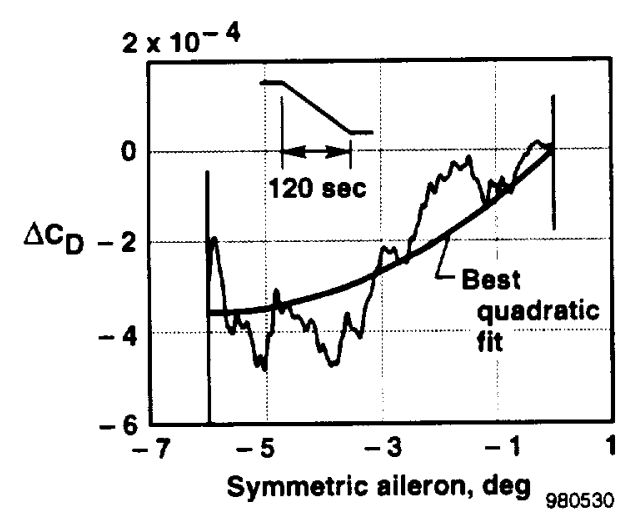

Figure 8. Variation of incremental drag with symmetric outboard aileron deflection for a ramp maneuver (data filtered, peak-to-peak excitation period $=120 \mathrm{sec}$, $\sigma=0.000093$ ).

The final $\Delta C_{D}$ estimation procedure, which was developed based on analysis of various maneuver types and maneuver parameters, is capable of resolving $\Delta C_{D}$ to approximately the one-drag-count level $(0.0001)$, which is consistent with the root mean squares of the analyses of figures 4,5 , and 8 . The average dragreduction benefit for the test aircraft, using only symmetric outboard ailerons, is between 2 and 3 drag counts, or nearly 1 percent of total aircraft drag.

\section{Real-Time Configuration Optimization}

The real-time research flight consisted of evaluating the performance of the adaptive configuration optimization algorithm at cruise flight conditions. The objectives of the flight analysis were to demonstrate and validate the operation of the algorithm for a range of maneuvers and maneuver variables. As discussed earlier, the test bed aircraft did not have a Mach-hold autothrottle system; maneuvers were performed both at constant throttle setting and with the pilot emulating a
Mach-hold mode by controlling Mach number with manual throttle control.

\section{Real-Time Optimization}

The real-time, in-flight adaptive configuration optimization process (fig. 9) consists of the following elements:

(a) Initiation of outboard aileron forced excitation.

(b) Storage of response data using a Ring-Buffered Network Bus (RBNB) TM (Creare, Inc., Hanover, New Hampshire). ${ }^{6}$

(c) Accessing data as required from the RBNBTM.

(d) Execution of the real-time optimization algorithm.

(e) Repetition of steps (b) to (d) and tracking the optimization results for convergence.

f) Upon convergence, commanding the outboard aileron to the optimally determined position.

Figure 10 shows a representative real-time optimization maneuver performed at Mach 0.84 and an altitude of $38,000 \mathrm{ft}$. The outboard aileron excitation period was $200 \mathrm{sec}$ with the excitation ranging from $-6.5^{\circ}$ (trailing edge down) to $6.5^{\circ}$ (trailing edge up) (note that the total excitation period is less than two periods, as discussed previously). Data from $2 \mathrm{~min}$ at the beginning and $1 \mathrm{~min}$ at the end of the maneuver, during which no excitation existed, were included in the analysis. The altitude-hold mode is constraining altitude to $\pm 20 \mathrm{ft}$ throughout the maneuver. The pilot controlled Mach number to approximately 0.004 peak-to-peak. The pilot control is reflected in the estimated thrust variation shown in the figure. This Mach number variation, which is very small, is also accounted for in the analysis as discussed previously.

Both the horizontal stabilator position and the trim angle of attack are controlled (directly and indirectly respectively) during the quasi-steady-state maneuver by the altitude-hold autopilot. The symmetric aileron illustrates both the forced-excitation maneuver and the application of the optimal (minimum drag) outboard aileron position. Figure 11 shows the analysis of the complete maneuver, which was performed in real time. The fit yields a minimum $\Delta C_{D}$ of approximately -0.0002 at $\delta_{a_{o p t}}=-3.4$; these results compare favorably with the results presented previously.

The optimization process described is anticipated to be functionally similar to a possible operational implementation. Additional details and considerations 


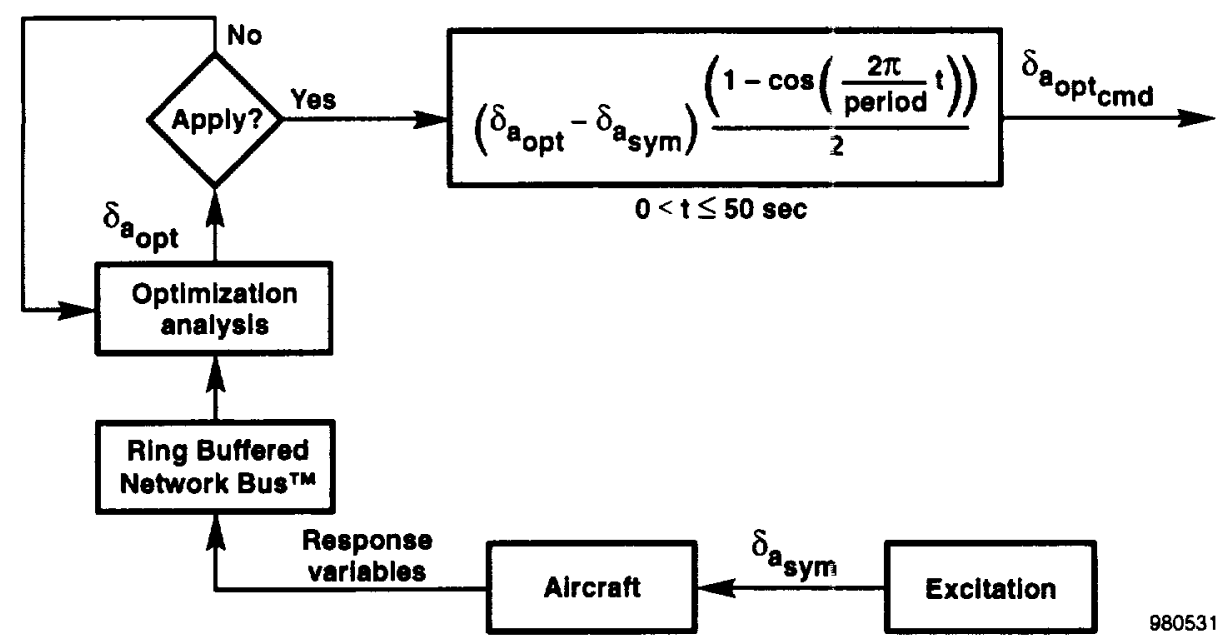

Figure 9. Block diagram of end-to-end optimization process.

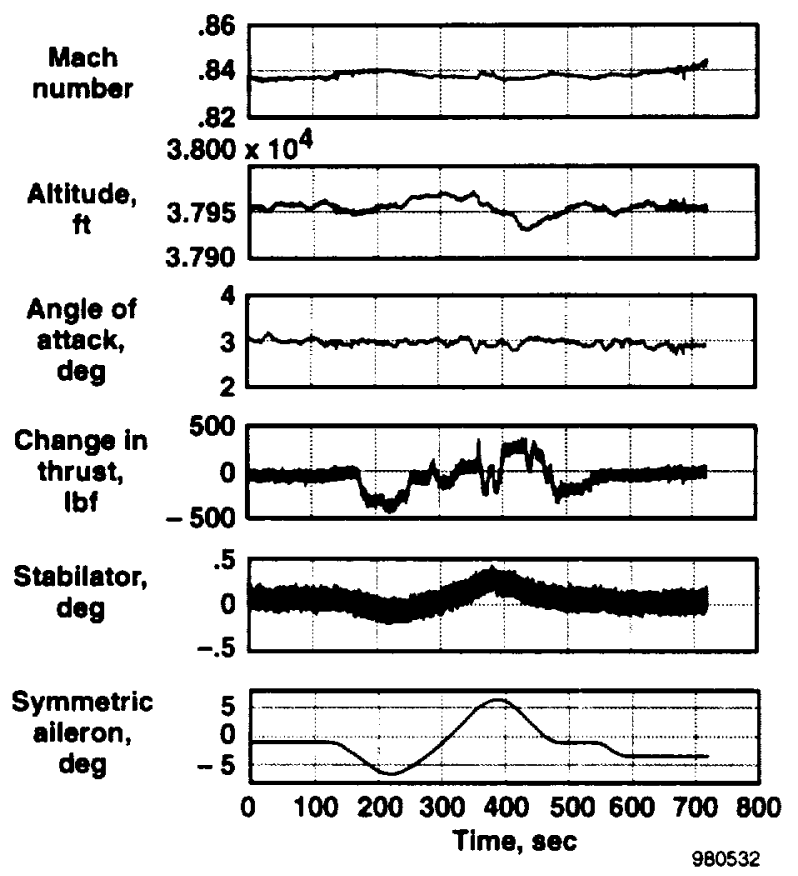

Figure 10. Flight response of an adaptive configuration optimization maneuver.

for implementation of an adaptive configuration optimization system in operational aircraft have previously been published. ${ }^{5}$

\section{Related Applications}

This paper considers explicit optimization of only one controller, symmetric outboard aileron position, because that was the only redundant controller available. The

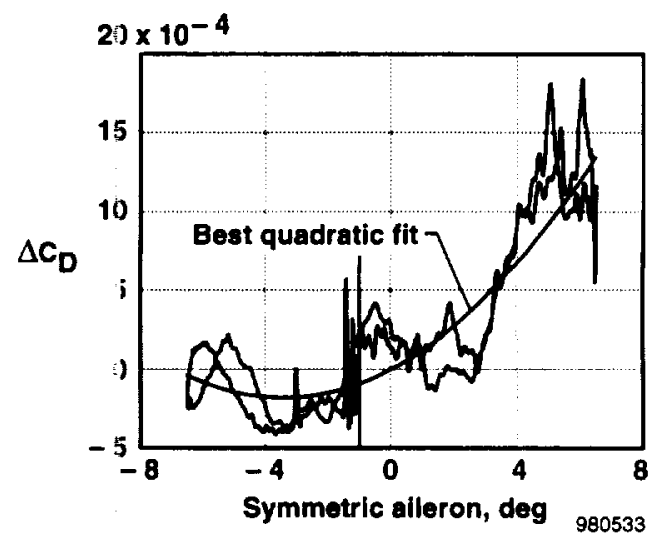

Figure 11. Variation of incremental drag with symmetric cutboard aileron deflection for a real-time adaptive cor figuration optimization maneuver; results obtained in eal time (data filtered, period $=200 \mathrm{sec}$, $\sigma=0.0003$ ).

technology lescribed and demonstrated is capable of discriminatiıg very small incremental drag values, and thus provide ; a very powerful optimization process for a wide range if applications. Some of these applications are discusserl below.

\section{Multisurface Application}

Future de: igns or modifications to current aircraft to incorporate :amber control of additional wing trailingedge surfaces could provide the opportunity for multisurface optimization. With this additional capability, ic eally each of the separate pairs of surfaces could be op imized independently. That is, if the wing 
had inboard and outboard ailerons and inboard and outboard flaps, the trailing-edge deflection for minimum drag would not be one constant deflection across the trailing edge but rather a variation from the inboardmost surface to the outboard-most surface. Because of cross-product terms, this multisurface optimization must be performed in an integrated manner, as opposed to just optimizing one pair of surfaces at a time then applying the four (in this case) sets of optimal positions. The same ideas can be applied to asymmetric optimization for the lateral-directional axes of the aircraft.

\section{Variable-Camber Wing}

All discussions to this point in time have considered using conventional control surfaces in a symmetric manner to optimize the configuration. Although use of existing trailing-edge control surfaces is technically a variable-camber capability, the use of the term "variable camber" is normally reserved for a specific capability designed into a configuration for the specific purpose of providing the same. The concept of true variable camber is ideal but also requires the configuration optimization capability (presented and discussed in this paper) to take full advantage of the capabilities.

\section{Close Formation Flight (Symmetric and Asymmetric Optimization)}

The concept of close formation flight to reduce drag, similar to the familiar vee formation birds use, is receiving attention in the aerodynamics community. Clearly, the interference aerodynamics of formation flight are complex and asymmetric for the general aircraft in the formation. A symmetric aircraft configuration would not be optimal in the minimum drag sense, and if redundant control surface (or variablecamber) capability existed, a more optimal configuration could be determined based on the principles previously presented in this paper. The adaptive configuration optimization process would not adversely affect the continuous control required to maintain the optimal formation positioning.

\section{High-Speed Civil Transport}

The High-Speed Civil Transport has the potential for accruing much larger benefits from the configuration optimization concepts discussed in this paper than from subsonic transports. The variable geometry of both the engine and inlets can be used in the optimization of propulsive thrust or net aircraft performance.

\section{Application to Drag Comparisons}

The incremental drag analysis of this paper is designed to identify incremental drag changes during a specific maneuver and is not designed for absolute drag analysis. However, the analysis approach described is suitable for making comparisons of one configuration to another, even from flight to flight. The only restriction would be that the measurement system could not have changed (for example, a measurable change occurring in a measurement bias). This capability has been demonstrated by comparing results obtained from absolute drag analysis of maneuvers designed for that purpose with results of the technique described in this paper using transient maneuvers.

\section{Concluding Remarks}

The NASA adaptive performance optimization flight research program has demonstrated the practical application of in-flight, real-time, adaptive configuration optimization for performance enhancement. The research flights were conducted on an L-1011 widebody transport that was modified to incorporate symmetric deflection of the outboard ailerons, which provided variable-camber capability. Explicit excitation of the redundant control surface (symmetric outboard aileron) explored variations of raised-cosine, two-sided raised-cosine, and ramp maneuvers. The dragminimization algorithm was shown to be capable of identifying drag to approximately the one-drag-count level.

On the L-1011 test bed aircraft, the net benefit of optimizing the symmetric outboard aileron position is a drag reduction of 2-3 drag counts (approximately 1 percent). Note that the outboard aileron represents a small portion (approximately 23-percent span from wing root to tip and approximately 3 percent of the wing area from wing root to tip) of the total wing trailingedge control potential. Many opportunities exist for application of the adaptive performance optimization methodology to current and future commercial and military transports. The optimization analysis algorithm can be implemented on commercial aircraft that have late-generation inertial navigation systems and airdata systems; engine pressure ratio measurements would be optional.

Algorithm analysis variations of various maneuver types indicate the following:

- A two-sided raised-cosine maneuver provides for improved definition of the quadratic representation 
of drag due to symmetric outboard aileron deflection in the optimization analysis and, therefore, provides more consistent results than one-sided raised-cosine maneuvers.

- The two-sided raised-cosine maneuver allows for a wide range in maneuver periods while producing satisfactory results.

- Ramp maneuvers have the favorable attribute of providing more even distribution of data collection as a function of excitation deffection than either type of raised-cosine maneuver.

- Although low-pass data filtering is required to produce visually acceptable results, the filtering does not noticeably affect the optimization algorithm results.

\section{$\underline{\text { References }}$}

'Gallagher, Thomas J., "Aircraft Finance and Airline Financial Analysis in the Fifth Cycle of the Jet Age," Handbook of Airline Economics, 1995, pp. 223- 234.
${ }^{2}$ Advance! Fighter Technology Integration F-111 Mission Adcptive Wing, NASA CP-3055, 1990 (ITAR restricted do sument).

${ }^{3}$ Bauer, C aus, et al., "A New Structural Approach to Variable Camber Wing Technology of Transport Aircraft," AlAA-98-1756, 1998.

${ }^{4}$ Gilyard, Glenn, Development of a Real-Time Transport Performance Optimization Methodology, NASA TM- $4730,1996$.

${ }^{5}$ Gilyard, Glenn, In-Flight Transport Performance Optimization: An Experimental Flight Research Program and an Operational Scenario, NASA TM-97-206229, 1997.

${ }^{6}$ Miller, Matthew J., et al., "On-Line Test and Measurement in a Distributed Collaborative Environmert," Proceedings of the Society for Experimental Mechanics, Inc., vol. 2, 1998, pp. 1313-1:319. 